\title{
Perfil epidemiológico das fraturas radiais distais em hospital de referência em Ribeirão Preto, Brasil
}

Epidemiological profile of distal radial fractures in a reference hospital in Ribeirão Preto, Brazil

Perfil epidemiológico de fracturas radiales distales en un hospital de referencia en Ribeirão Preto, Brasil

Felipe Azevedo Mendes de OLIVEIRA ${ }^{1}$

Thiago Agostini Pereira ALBENY2

Luis Guilherme Rosifini Alves REZENDE ${ }^{3}$

Filipe Jun SHIMAOKA ${ }^{3}$

Amanda Favaro CAGNOLATI ${ }^{3}$

Aléx Eduardo Calderón IRUSTA ${ }^{3}$

Luiz Garcia MANDARANO-FILHO ${ }^{3}$

Nilton MAZZER

${ }^{I}$ Cirurgião de Mão, formado no programa de Cirurgia da Mão, Hospital das Clínicas, Faculdade de Medicina de Ribeirão Preto, Universidade de São Paulo (HC-FMRP-USP), Ribeirão Preto, SP, Brasil

${ }^{2}$ Cirurgião de Mão no Hospital Sírio Libanês (Brasília), formado no programa de Cirurgia da Mão, Hospital das Clínicas, Faculdade de Medicina de Ribeirão Preto, Universidade de São Paulo (HC-FMRP-USP), Ribeirão Preto, SP, Brasil

${ }^{3}$ Médico Assistente do Programa de Cirurgia da Mão, Hospital das Clínicas, Faculdade de Medicina de Ribeirão Preto, Universidade de São Paulo, (HC-FMRP-USP), Ribeirão Preto, SP, Brasil

${ }^{4}$ Professor Titular e Chefe da Divisão de Cirurgia da Mão, Hospital das Clínicas, Faculdade de Medicina de Ribeirão Preto, Universidade de São Paulo (HC-FMRP-USP), Ribeirão Preto, SP, Brasil

\section{Resumo}

Objetivo: Avaliar o perfil epidemiológico das fraturas do rádio distal em hospitais de referência em Ribeirão Preto(SP), Brasil. Não existem dados suficientes na literatura nacional que corroborem com o perfil epidemiológico das fraturas do rádio distal. Métodos: 245 pacientes apresentaram 254 fraturas do rádio distal, ocorridas entre 2014 a 2017 foram avaliadas retrospectivamente para obtenção do perfil epidemiológico. Os fatores analisados foram idade e sexo, mecanismo do trauma, sazonalidade, tipo de fratura baseada na Classificação AO, presença de exposição óssea, lesões associadas, tipo de tratamento realizado (conservador ou cirúrgico) e o tipo de implante utilizado nos tratamentos cirúrgicos. Resultados: 60,2\% dos pacientes participantes eram do sexo masculino e 39,8\% do sexo feminino, distribuídos de forma bimodal. A média de idade foi 45,4 anos. Fraturas expostas corresponderam a 92,1\% das fraturas e 7,9\% representaram as expostas. Pacientes politraumatizados representaram 62,6\%. O tempo médio de internação foi 8,09 dias. Conclusão: Apesar do padrão de fraturas mostrar semelhanças com outros estudos, o padrão apresentado pode não traduzir, de forma homogênea, o padrão obtido em outras metrópoles e grandes centros.

Descritores: Fraturas do Rádio; Traumatismos do Punho; Epidemiologia; Hospitais Especializados.

\section{Abstract}

Aim of Study: To evaluate the epidemiological profile of distal radius fractures in a reference hospital in Ribeirão Preto (SP), Brazil. There are not enough data in the national literature to corroborate the epidemiological profile of distal radius fractures. Methods: 245 patients had 254 fractures of the distal radius, which occurred between 2014 and 2017, were retrospectively evaluated to obtain the epidemiological profile. The factors analyzed were age and sex, trauma mechanism, seasonality, type of fracture based on the AO Classification, presence of bone exposure, associated injuries, type of treatment performed (conservative or surgical) and the type of implant used in surgical treatments. Results: $60.2 \%$ of the participating patients were male and $39.8 \%$ female, distributed in a bimodal manner. The average age was 45.4 years. Open fractures corresponded to $92.1 \%$ of fractures and $7.9 \%$ represented exposed ones. Polytrauma patients accounted for $62.6 \%$. The average hospital stay was 8.09 days. Conclusion: Although the fracture pattern shows similarities with other studies, the pattern presented may not translate, in a homogeneous way, the pattern obtained in other metropolises and large centers.

Descriptors: Radius Fractures; Wrist Injuries; Epidemiology; Hospitals, Special.

\section{Resumen}

Objetivo: Evaluar el perfil epidemiológico de las fracturas de radio distal en hospitales de referencia de Ribeirão Preto (SP), Brasil. No existen datos suficientes en la literatura nacional para corroborar el perfil epidemiológico de las fracturas de radio distal. Métodos: 245 pacientes que presentaban 254 fracturas de radio distal, ocurridas entre 2014 y 2017, fueron evaluadas retrospectivamente para obtener el perfil epidemiológico. Los factores analizados fueron edad y sexo, mecanismo de traumatismo, estacionalidad, tipo de fractura según la Clasificación AO, presencia de exposición ósea, lesiones asociadas, tipo de tratamiento realizado (conservador o quirúrgico) y tipo de implante utilizado en los tratamientos quirúrgicos. Resultados: El 60,2\% de los pacientes participantes fueron varones y el $39,8 \%$ mujeres, distribuidos de forma bimodal. La edad media fue de 45,4 años. Las fracturas abiertas correspondieron al 92,1\% de las fracturas y el 7,9\% representaron las expuestas. Los pacientes politraumatizados representaron el 62,6\%. La estancia hospitalaria media fue de 8,09 días. Conclusión: Aunque el patrón de fractura muestra similitudes con otros estudios, el patrón presentado puede no traducir, de manera homogénea, el patrón obtenido en otras metrópolis y grandes centros.

Descriptores: Fracturas del Radio; Traumatismo de la Muñeca; Epidemiología; Hospitales Especializados.

\section{INTRODUÇÃO}

A fratura da extremidade distal do rádio é uma lesão frequente. Nos Estados Unidos, o número de casos ultrapassou 640 mil por ano e representou $16 \%$ a $20 \%$ de todos os atendimentos de urgência, expressando $26 \%$ a $46 \%$ do total das fraturas ${ }^{1,2}$. Elas também representaram $17,5 \%$ do total de fraturas em adultos em Edimburgo ${ }^{3}$. Correspondem a $44 \%$ das fraturas na Suécia, sendo $2,5 \%$ do total dos atendimentos de urgência ${ }^{4}$.

Sabe-se que a distribuição relativa à idade e sexo tem característica bimodal, cujo predomínio de fraturas de alta energia em adultos jovens do sexo masculino é influenciado pela prática de atividades esportivas, queda de mais de $1 \mathrm{~m}$ de altura, acidente de veículos automotores ou bicicleta ${ }^{6}$. Fraturas de baixa energia resultam principalmente da queda de própria altura com a mão espalmada em idosas ${ }^{4,5,9,11}$. Predomina no inverno em países escandinavos, através de quedas em jovens do sexo masculino durante esportes de inverno ou pelo escorregamento de idosas em pisos cobertos por neve $\mathrm{e}^{5-11}$. Autores orientais relataram uma maior incidência dessas fraturas no verão devido à alta ocorrência de tufões $^{2,12-14}$. 
A maioria dos estudos traduz que a incidência destas fraturas vem aumentando nos últimos 50 anos, com variável estabilização em valores mais elevados, nas duas últimas décadas ${ }^{2,4-6,8,10,15}$. Observam também algumas peculiaridades na distribuição por idade e sexo, como a presença de dois picos de incidência em idosos $^{8,10,15}$. O primeiro, ocorreu em mulheres pósmenopausa (50-60 anos); e o segundo, em idosos acima de 90 anos, independente do sexo ${ }^{6,8,10,15}$.

Poucos dados encontram-se disponíveis em relação ao estudo epidemiológico no Brasil. O objetivo deste trabalho é analisar as características epidemiológicas dos indivíduos e das lesões nos portadores de fraturas da extremidade distal do rádio, atendidos no complexo do Hospital das Clínicas da Faculdade de Medicina de Ribeirão Preto (HCFMRPUSP).

\section{MATERIAL E MÉTODO}

Trata-se de estudo retrospectivo realizado no Hospital das Clínicas da Faculdade de Medicina de Ribeirão Preto da Universidade de São Paulo (USP) em Ribeirão Preto (SP), Brasil, e aprovado pelo Comitê de Ética em Pesquisa do Hospital das Clínicas da Faculdade de Medicina de Ribeirão Preto.

Foram analisados prontuários de 245 pacientes esqueleticamente maduros e com fratura da extremidade distal do rádio, atendidos entre 2014 e 2017. Os fatores avaliados incluíram idade e sexo, mecanismo do trauma, sazonalidade, tipo de fratura baseada na Classificação AO (Arbeitsgemeinschaft für Osteosynthesefragen), presença de exposição óssea, lesões associadas, tipo de tratamento realizado (conservador ou cirúrgico) e o tipo de implante utilizado nos tratamentos cirúrgicos. Também foram analisados o intervalo de tempo entre o trauma e o tratamento definitivo; e o tempo de internação hospitalar. Os dados coletados receberam tratamento estatístico descritivo, empregando-se valores numéricos para os dados quantitativos e de frequência para os qualitativos ${ }^{16}$.

\section{RESULTADOS}

Dentre as 254 fraturas analisadas, 153 $(60,2 \%)$ eram em pacientes do sexo masculino e 101 $(39,8 \%)$ em pacientes do sexo feminino. A média de idade dos pacientes foi de 45,4 anos, variando de 18 a 87 anos. O gráfico da Figura 1 mostra a distribuição de idade e sexo dos pacientes. O tipo de trauma predominante foi acidente de trânsito por motociclismo. A distribuição do mecanismo de trauma pode ser observada na Tabela 1.

A correlação entre a faixa etária do paciente e o mecanismo do trauma encontra-se disponível na Figura 2. Considerando o padrão de sazonalidade, observa-se $29,5 \%$ no inverno, $28,8 \%$ no outono, $22,8 \%$ na primavera e $19,7 \%$ no verão, sem diferença estatística entre os padrões.
Tabela 1. Mecanismo de trauma, número de casos e porcentagem total \begin{tabular}{|l|l|l|}
\hline Tipo de Trauma & (n) & \% do Total \\
\hline
\end{tabular} \begin{tabular}{|l|l|l|}
\hline Motociclismo & 89 & $35,1 \%$ \\
\hline
\end{tabular} \begin{tabular}{|l|l|l|}
\hline Queda de Própria Altura & 79 & $31,1 \%$ \\
\hline Quedas (Alturas, Bicicleta, Cavalo, Escada) & 60 & $23,6 \%$ \\
\hline
\end{tabular} \begin{tabular}{lll} 
Quedas (Alturas, Bicicleta, Cavalo, Escada) & 60 & $23,6 \%$ \\
\hline Automobilístico &
\end{tabular} \begin{tabular}{|l|l|l}
\hline Diversas (TD, FAB, FAF, Mordidas animais, & 15 & $5,9 \%$
\end{tabular} \begin{tabular}{l}
$\begin{array}{l}\text { Diversas (TD, FAB, FAF, Mordidas animais, } \\
\text { Espancamento) }\end{array}$ \\
\hline
\end{tabular} TOTAL \begin{tabular}{l|l}
254 & $100 \%$ \\
\hline
\end{tabular} *TD: trauma direto; FAB: ferimento por arma branca; FAF: ferimento por arma de fogo Fonte: Dados da Pesquisa

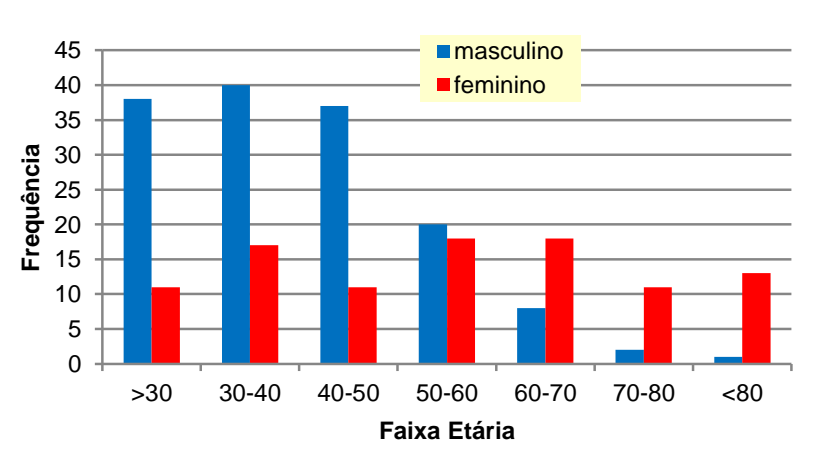

Figura 1. Relação do gênero e faixa etária (Fonte: Dados da Pesquisa)

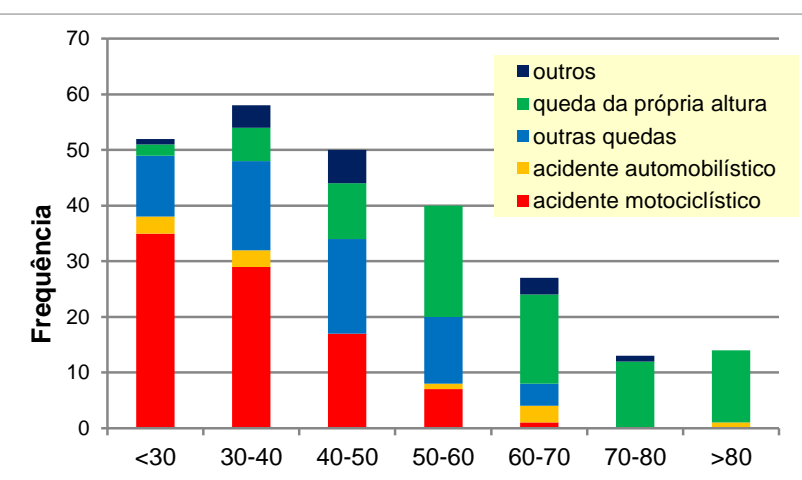

Figura 2: Relação do tipo de trauma e faixa etária (Fonte: Dados da Pesquisa).

A distribuição das fraturas de acordo com a Classificação AO encontra-se disponível na Tabela 2. A relação entre o tipo de fratura na classificação $\mathrm{AO}$ e o mecanismo do trauma $(\mathrm{p}=0,003509)$ estão apresentados na Figura 3.

Tabela 2. Classificação AO (Tipo 23) e Epidemiologia

\begin{tabular}{l|l|l}
\hline Tipo & (n) & \% \\
\hline A2 & $\mathbf{2 2}$ & 8,7 \\
\hline A2 & 21 & 8,3 \\
\hline B1 & 25 & 9,8 \\
\hline B2 & 8 & 3,2 \\
\hline B3 & 19 & 7,5 \\
\hline C1 & 42 & 16,5 \\
\hline C2 & 28 & 11 \\
\hline C3 & 89 & 35 \\
\hline TOTAL & 254 & $100 \%$ \\
\hline \multicolumn{2}{l}{ Fonte: Dados da Pesquisa } &
\end{tabular}

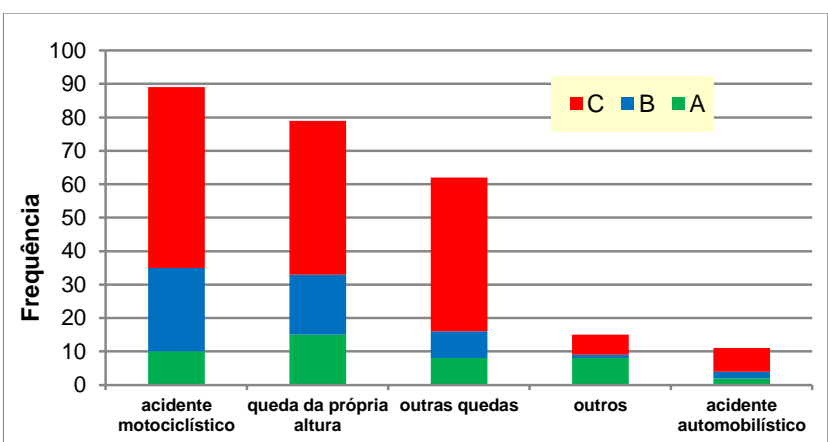

Figura 3: Relação do tipo de trauma e Classificação AO (Fonte: Dados da Pesquisa). 
Considerando a exposição óssea, $92,1 \%$ das fraturas foram fechadas e 7,9\% apresentaram exposição óssea. Politraumatizados representaram $62,6 \%$ dos casos. Dentre os politraumatizados, $44,5 \%$ apresentavam lesões no punho e mão ipsilateral e 7,5\% apresentavam alguma lesão no membro superior ipsilateral. Lesões ortopédicas distantes do membro ipsilateral representaram $21,7 \%$. Pacientes com outras lesões não ortopédicas representaram 5,5\% dos casos. Em relação ao tratamento cirúrgico, $25,2 \%$ foram tratadas conservadoramente e $74,8 \%$ cirurgicamente. A relação do tipo de tratamento (conservador ou cirúrgico) e a faixa etária podem ser observadas na Figura 4, enquanto a relação entre o tipo de tratamento e a Classificação $\mathrm{AO}$ estão demonstradas na Figura 5. O tipo de implante utilizado e sua relação com a Classificação AO encontra-se disponível na Tabela 3.

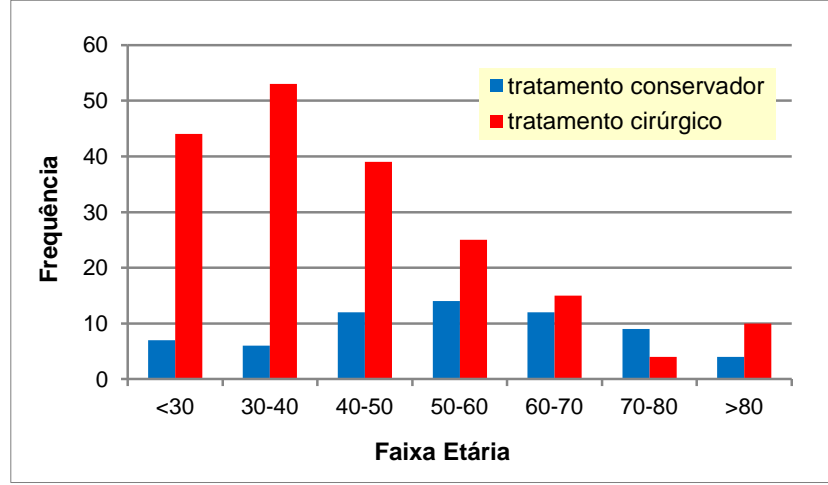

Figura 4: Relação do tipo de tratamento e faixa etária (Fonte: Dados da Pesquisa).

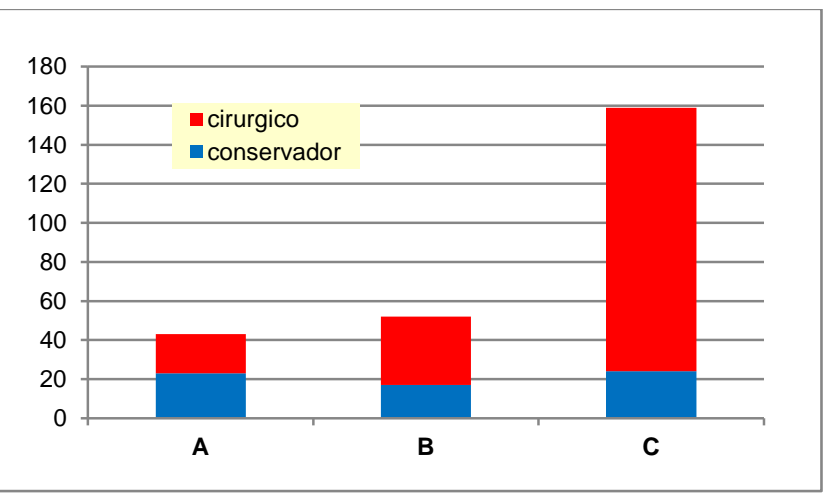

Figura 5: Relação do tipo de tratamento e padrão da fratura (Fonte: Dados da Pesquisa).

Tabela 3. Relação entre método de fixação e Classificação AO

\begin{tabular}{l|l|l|l}
\hline & A & B & C \\
\hline FK & $5 \%$ & $8,6 \%$ & $3,7 \%$ \\
\hline FE Transarticular & 0 & 0 & $2,2 \%$ \\
\hline FE + FK & 0 & $2,8 \%$ & $5,2 \%$ \\
\hline Parafuso canulado & $5 \%$ & $20 \%$ & $0,7 \%$ \\
\hline Placa Volar & $85 \%$ & $51,4 \%$ & $63,1 \%$ \\
\hline Placa Dorsal & 0 & $8,6 \%$ & $0,7 \%$ \\
\hline Placa Volar + Dorsal & $5 \%$ & $8,6 \%$ & $24,4 \%$ \\
\hline TOTAL POR GRUPO & $100 \%$ & $100 \%$ & $100 \%$ \\
*FK: fio de Kirschner; FE: fixador externo \\
Fonte: Dados da Pesquisa
\end{tabular}

A média do intervalo de tempo decorrido entre o trauma e o tratamento definitivo foi de 8,09 dias (1 a 90 dias). Pacientes com lesão associada aguardaram em média 7,79 dias (entre 1 e 38 dias) e os sem lesão associada, em média por 8,59 dias (1 a
90). O tempo de internação médio foi de 8,19 dias ( 0 a 67); cujo tempo médio foi de 5,31 dias ( 0 a 35) para pacientes sem lesões, enquanto os com lesões tiveram média de 9,92 dias (0 a 67) com diferença estatística entre eles $(\mathrm{p}<0,01)$.

\section{DISCUSSÃO}

Também observamos o mesmo padrão epidemiológico bimodal observado em outros estudos $^{2,3-6,9,11,13}$. Court-Brown ${ }^{17}$ obteve uma média de idade de 58,4 anos, sendo $18,1 \%$ de pacientes maiores de 80 anos e a relação entre o sexo masculino/feminino (M/F) de 28/7217; eles também obtiveram média de idade de 45,4 anos e $5,5 \%$ de pacientes acima de 80 anos com relação $\mathrm{M} / \mathrm{F}$ de 60/4017. Embora tenham categorizado os mecanismos de trauma de forma diferente, observa-se uma prevalência muito grande de queda da própria altura que, neste trabalho não representou a principal causa dos traumas ${ }^{17}$. Isto se deve ao fato de este estudo ser realizado num hospital de referência (nível terciário), onde o padrão de trauma predominante é o de alta energia, o que hipoteticamente justifica a predominância de acidentes por motociclismo, onde as quedas de própria altura apresentaram o segundo mecanismo de trauma mais comum. Queda de própria altura foi a segunda causa mais comum, principalmente em idosas ${ }^{2,6}$.

Note-se ainda que, segundo Flinkkila et al. ${ }^{5}$ traumas de alta energia em ossos normais ou de baixa energia em ossos osteoporóticos podem resultar em fraturas similares, resultado também observado neste trabalho. Não observamos diferenças estatisticamente significativas em relação à sazonalidade. A fratura mais encontrada foi a do Tipo $\mathrm{C} \quad(62,6 \%)$, contrastando com o padrão do Tipo $\mathrm{A}$, mais comum nos demais estudos ${ }^{9,11,13}$. O padrão do tipo $\mathrm{C}$ aumenta conforme o aumento da energia do trauma ${ }^{14}$.

Court-Brown ${ }^{17}$, em análise das fraturas expostas, encontrou $7,7 \%$ delas, média de idade dos pacientes de 41,9 anos, 1,8\% maiores de 80 anos e relação $\mathrm{M} / \mathrm{F}$ de 66/34. Neste trabalho encontramos $7,9 \%$ de fraturas expostas, média de idade de 46,5 anos, $10 \%$ de pacientes maiores de 80 anos e relação $\mathrm{M} / \mathrm{F}$ de 55/45. Observe-se que, embora a incidência de fraturas expostas seja praticamente a mesma, mulheres em idade imediatamente pós-menopausa foram responsáveis pelo aumento da média de idade e diminuição da relação M/F.

São escassos os trabalhos na literatura que incluem as lesões associadas às fraturas da extremidade distal do rádio. Koo et al. ${ }^{13}$ observaram $41,7 \%$ de lesões ósseas no membro superior ipsilateral à fratura; nosso estudo observou $52 \%$ de lesões ortopédicas associadas à fratura no mesmo membro. Nosso número pode ter sido superior por termos incluído todas as lesões ortopédicas, e não apenas as ósseas. 
Estudos apontam que $40 \%$ destas fraturas são tratadas de forma cirúrgica ${ }^{5}$. Entretanto, até $80 \%$ dos casos são tratados de forma conservadora ${ }^{18}$.

A predominância pela placa volar também foi observada em outros estudos ${ }^{5,19}$. Também observamos decréscimo na proporção de tratamento cirúrgico conforme aumento da idade, também observado em outros estudos ${ }^{14}$. Este fato também é contrastado em outros estudos, como o de Lindau e Arner $^{6}$ que não apresentou diferença na proporção de tratamento cirúrgico em relação à faixa etária9. Observamos uma predominância de tratamento cirúrgico, conforme o aumento da energia do trauma, conforme observado em outros estudos ${ }^{14}$.

A relação entre o tempo médio entre admissão e tratamento definitivo foi de 8,09 dias. Os pacientes com lesões associadas demandaram maior tempo de internação, devido lesões associadas ou a necessidade de tratamento estagiado.

\section{CONCLUSÃO}

Apesar de observamos padrão de distribuição bimodal entre idade e sexo com uma relação entre a magnitude da energia do trauma, mais estudos são necessários, considerando o padrão epidemiológico do Brasil. Este estudo expressa o padrão epidemiológico de um hospital terciário em Ribeirão Preto-SP, podendo não traduzir a realidade de outras regiões metropolitanas, de acordo com a alta variabilidade sociocultural e econômica do Brasil.

\section{REFERÊNCIAS}

1. Bruce KK, Merenstein DJ, Narvaez MV, Neufeld SK, Paulus MJ, Tan TP et al. Lack of Agreement on Distal Radius Fracture Treatment. J Am Board Fam Med. 2016;29(2):218-25.

2. MacIntyre NJ, Dewan N. Epidemiology of distal radius fractures and factors predicting risk and prognosis. J Hand Ther. 2016;29(2):136-45.

3. Court-Brown CM, Caesar B. Epidemiology of adult fractures: A review. Injury. 2006; 37(8):691-97.

4. Nellans KW, Kowalski E, Chung KC. The epidemiology of distal radius fractures. Hand Clin. 2012;28(2):113-25.

5. Flinkkilä T, Sirniö K, Hippi M, Hartonen S, Ruuhela R, Ohtonen P et al. Epidemiology and seasonal variation of distal radius fractures in Oulu, Finland. Osteoporos Int. 2011; 22(8):2307-312.

6. Lindau TR, Aspenberg P, Arner M, RedlundhJohnell I, Hagberg L. Fractures of the distal forearm in young adults. An epidemiologic description of 341 patients. Acta Orthop Scand. 1999;70(2):124-28.

7. Diamantopoulos AP, Rohde G, Johnsrud I, Skoie IM, Hochberg M, Haugeberg G. The epidemiology of low- and high-energy distal radius fracture in middle-aged and elderly men and women in Southern Norway. PLoS One. 2012;7(8):e43367.

8. Wilcke MK, Hammarberg H, Adolphson PY. Epidemiology and changed surgical treatment methods for fractures of the distal radius: a registry analysis of 42,583 patients in Stockholm County, Sweden, 2004-2010. Acta Orthop. 2013;84(3):292-96.

9. Sigurdardottir K, Halldorsson S, Robertsson J. Epidemiology and treatment of distal radius fractures in Reykjavik, Iceland, in 2004. Comparison with an Icelandic study from 1985. Acta Orthop. 2011;82(4):494-98.

10. Solgaard S, Petersen VS. Epidemiology of distal radius fractures. Acta Orthop Scand. 1985;56(5):391-93.

11. Brogren E, Petranek M, Atroshi I. Incidence and characteristics of distal radius fractures in a southern Swedish region. BMC Musculoskelet Disord. 2007;8:48.

12. Tsai $\mathrm{CH}$, Muo $\mathrm{CH}$, Fong YC, et al. A populationbased study on trend in incidence of distal radial fractures in adults in Taiwan in 20002007. Osteoporos Int. 2011;22(11):2809-815.

13. Koo OT, Tan DM, Chong AK. Distal radius fractures: an epidemiological review. Orthop Surg. 2013;5(3):209-13.

14. Dóczi J, Renner A. Epidemiology of distal radius fractures in Budapest. A retrospective study of 2,241 cases in 1989. Acta Orthop Scand. 1994;65(4):432-33.

15. Chen NC, Jupiter JB. Management of distal radial fractures. J Bone Joint Surg Am. 2007; 89(9):2051-62.

16. Pagano M, Gauvreau K. Princípios de Bioestatística. 2. ed. São Paulo: Pioneira Thompson Learning; 2004.

17. Court-Brown CM. Epidemiologia das fraturas e luxações. In: Court-Brown CM et al. (ed.); Fraturas em adultos de Rockwood \& Green. 8. ed. Barueri, SP: Manole; 2016.

18. Fanuele J, Koval KJ, Lurie J, Zhou W, Tosteson A, Ring D. Distal radial fracture treatment: what you get may depend on your age and address. J Bone Joint Surg Am. 2009;91(6):1313-19.

19. Jupiter JB, Marent-Huber M; LCP Study Group. Operative management of distal radial fractures with 2.4-millimeter locking plates: a multicenter prospective case series. Surgical technique. J Bone Joint Surg Am. 2010; 92(Suppl 1 Pt1):96-106. 


\section{CONFLITO DE INTERESSES}

Os autores declaram não haver conflitos de interesse.

\section{AUTOR PARA CORRESPONDÊNCIA}

\section{Luis Guilherme Rosifini Alves Rezende}

Hospital das Clínicas da Faculdade de Medicina de Ribeirão

Preto da Universidade de São Paulo (USP)

Av. Bandeirantes, 3900 - Vila Monte Alegre

14049-900 Ribeirão Preto - SP, Brasil

Email: lgrezende@usp.br

Submetido em 16/05/2020

Aceito em 28/06/2020 